\title{
Phenotype and molecular characterizations of a family with dentinogenesis imperfecta shields type II with a novel DSPP mutation
}

\author{
Qin Du ${ }^{1,2,3 \#}$, Li Cao ${ }^{2 \#}$, Yi Liu ${ }^{1}$, Chunyan Pang ${ }^{1}$, Si Wu ${ }^{4}$, Liwei Zheng ${ }^{4}$, Wei Jiang ${ }^{2}$, Xiaoxue $\mathrm{Na}^{2}, \mathrm{Jing} \mathrm{Yu}^{2}$, \\ Shasha Wang ${ }^{1}$, Xianjun $\mathrm{Zhu}^{2}$, Jiyun Yang ${ }^{2}$ \\ ${ }^{1}$ Department of Stomatology, Sichuan Provincial People's Hospital, University of Electronic Science and Technology of China, Chengdu, China; \\ ${ }^{2}$ The Key Laboratory for Human Disease Gene Study of Sichuan Province and Prenatal Diagnosis Center, Sichuan Provincial People's Hospital, \\ University of Electronic Science and Technology of China, Chengdu, China; ${ }^{3}$ Chengdu Institute of Biology, Chinese Academy of Sciences, Chengdu, \\ China; ${ }^{4}$ The State Key Lab of Oral Diseases, West China Hospital of Stomatology, Sichuan University, Chengdu, China \\ Contributions: (I) Conception and design: J Yang, X Zhu; (II) Administrative support: J Yang; (III) Provision of study materials or patients: Q Du; (IV) \\ Collection and assembly of data: L Cao, Y Liu, C Pang, W Jiang; (V) Data analysis and interpretation: S Wu, S Wang, L Zheng, X Na, J Yu; (VI) \\ Manuscript writing: All authors; (VII) Final approval of manuscript: All authors. \\ "These authors contributed equally to this work. \\ Correspondence to: Jiyun Yang; Xianjun Zhu. The Key Laboratory for Human Disease Gene Study of Sichuan Province and Prenatal Diagnosis Center, \\ Sichuan Provincial People's Hospital, University of Electronic Science and Technology of China, 32, Section 2, The First Ring Road West, Chengdu \\ 610072, China. Email: yangjiyun@yeah.net; xjzhu@uestc.edu.cn.
}

Background: Dentinogenesis imperfecta (DGI), Shields type-II is an autosomal dominant genetic disease which severely affects the function of the patients' teeth. The dentin sialophosphoprotein (DSPP) gene is considered to be the pathogenic gene of DGI-II. In this study, a DGI-II family with a novel DSPP mutation were collected, functional characteristics of DGI cells and clinical features were analyzed to better understand the genotype-phenotype relationship of this disease.

Methods: Clinical data were collected, whole exome sequencing (WES) was conducted, and Sanger sequencing was used to verify the mutation sites. Physical characteristics of the patient's teeth were examined using scanning electron microscopy (SEM) and energy dispersive spectroscopy (EDS). The localization of green fluorescent protein (GFP)-fused wild-type (WT) dentin sialoprotein (DSP) and its variant were evaluated via an immunocytochemistry (ICC) assay. The behaviors of human dental pulp stem cells (hDPSCs) were investigated by flow cytometry, osteogenic differentiation, and quantitative real-time polymerase chain reaction (qRT-PCR).

Results: A novel heterozygous mutation c.53T > G (p. Val18Gly) in DSPP was found in this family. The SEM results showed that the participants' teeth had reduced and irregular dentinal tubes. The EDS results showed that the $\mathrm{Ca} / \mathrm{P}$ ratio of the patients' teeth was significantly higher than that of the control group. The ICC assay showed that the mutant DSP was entrapped in the endoplasmic reticulum (ER), while the WT DSP located mainly in the Golgi apparatus. In comparison with normal cells, the patient's cells exhibited significantly decreased mineralization ability and lower expression levels of DSPP and RUNX2.

Conclusions: The c.53T > G (p. Val18Gly) DSPP variant was shown to present with rare hypoplastic enamel defects. Functional analysis revealed that this novel variant disturbs dentinal characteristics and pulp cell behavior.

Keywords: Dentinogenesis imperfecta (DGI); Shields type-II; dentin sialophosphoprotein (DSPP); dental pulp stem cells (DPSCs) 
Submitted Sep 14, 2021. Accepted for publication Nov 09, 2021.

doi: 10.21037/atm-21-5369

View this article at: https://dx.doi.org/10.21037/atm-21-5369

\section{Introduction}

Hereditary dentin disorders are a group of rare autosomal dominant genetic diseases characterized by dentin formation defects, which lead to the early loss of overlying enamel and a high risk of dental caries and early tooth loss $(1,2)$. These disorders include dentinogenesis imperfecta (DGI) and dentin dysplasia (DD). On the basis of clinical manifestations, DGI is classified into 3 types, and DD is classified into 2 types by Shields (3). Of the types, DGI, Shields type II affects approximately 1/6,000-1/8,000 of the population (4) and manifests primary as amber browncolored teeth, early pulp canal obliteration by dentinal tissue, and severe abrasion of both primary and permanent teeth (5). The primary dentition is usually the most severely affected (6). Additionally, DGI, Shields type II leads to early exfoliation of dental enamel and severe caries, periapical abscesses, and early tooth loss, affecting patients' chewing, facial shape, and systemic nutrition. Besides teeth symptoms, some patients may also suffer from hearing loss. DGI may causes psychological problems in patients and their families, such as feelings of inferiority and depression (7).

The dentin sialophosphoprotein $(D S P P)$ gene is located on chromosome $4 \mathrm{q} 21.3$, and it has 5 exons. The DSPP gene encodes a member of the small integrin-binding ligand $\mathrm{N}$-linked glycoprotein (SIBLING) family of proteins. The encoded preproprotein is secreted by odontoblasts and proteolytically processed to generate two principal proteins, dentin sialoprotein (DSP) and dentin phosphoprotein (DPP). DSP and DPP may play different roles in dentin mineralization. DSP may participate in dentinogenesis. DPP may participate in the initial mineralization of dentin matrix $(8,9)$. The DSPP gene is the sole pathogenic gene of DGI, shields type II (OMIM 125490), DGI, shields type III (OMIM 125500), DD, shields type II (OMIM 125420), and deafness with dentinogenesis (OMIM 605594) (10-13). To date, more than 50 heterozygous mutations of DSPP (NM_014208.3) have been identified in patients with these diseases. However, the relationship of the phenotype and genotype is remained to be elucidated.

In this study, we report the finding for a family with DGI, shields type II. Individuals in this family also exhibit primary enamel defects. Whole exome sequencing (WES) revealed a novel heterozygous mutation c.53T $>$ G (p. Val18Gly) of DSPP in the proband. The variant co-segregated with the disease phenotype in the family under study. Functional analyses revealed that this novel variant impairs DSPP function. This paper aimed to provide valuable information on disease pathogenesis and yield a better understanding of the genotype-phenotype relationship. We present the following article in accordance with the MDAR reporting checklist (available at https:// dx.doi.org/10.21037/atm-21-5369).

\section{Methods}

\section{Participants and clinical assessments}

All procedures involving human participants in this study were performed in accordance with the ethical standards of the Medical Ethics Review Committee of Sichuan Provincial People's Hospital and the Declaration of Helsinki (as revised in 2013). All participants provided written informed consent. This study was approved by the Institutional Review Board of the Sichuan Provincial People's Hospital [No. 2020(02)].

A 4-generation DGI family with 4 affected individuals was enrolled in this study. The proband (female, $7 \mathrm{y}$ ) and her parents (III:1, III:2, and IV:1) (father, $40 \mathrm{y}$; mother 36 y) were recruited, and all underwent complete dental assessment, including oral panoramic radiographs and cone-beam computed tomography (CBCT). No symptoms of hearing loss or bone defects were detected in 2 of the participants.

\section{DNA extraction}

Blood samples of the proband and her parents (III:1, III:2, and IV:1) were also collected. Genomic DNA was isolated using the TIANGEN Blood DNA Kit (TIANGEN, Beijing, China) in accordance with the standard protocol. The DNA samples were stored at the temperature of $-20{ }^{\circ} \mathrm{C}$ until use, and DNA integrity was assessed by $1 \%$ agarose gel electrophoresis. 


\section{WES and mutation analysis}

We performed WES of the proband's genomic DNA samples (IV:1) via MyGenostics Technology, Inc. (Beijing, China). Analysis of WES data and annotation of variants were carried out as previously reported (14). Briefly, WES raw data were processed by Real Time Analysis (RTA) software using default parameters. The sequencing reads were aligned to the human reference genome (hg19) using Burrows-Wheeler Aligner (BWA) software (https:// bio-bwa.sourceforge.net/bwa.shtml). The variants were annotated and filtered against the following public databases and in-house database: dbSNP138 (https://www.ncbi.nlm. nih.gov/projects/SNP/), 1000 Genomes Project (ftp:// ftp.1000genomes.ebi.ac.uk/vol1/ftp), Gnomad (https:// gnomad.broadinstitute.org/), and our in-house database generated from 1,977 WES samples. Common variants (minor allele frequency, $>0.1 \%$ ) were excluded. Variants were classified in accordance with the interpretation guidelines of the American College of Medical Genetics and Genomics (ACMG), as potentially pathogenic variants, variants of unknown clinical significance, or benign variants (15). Deleterious variants of unknown clinical significance were further classified as associated or not associated to the proband phenotype. In this study, we focused on disease-causing genes for DGI, osteogenesis imperfecta, and amelogenesis imperfecta in the Online Mendelian Inheritance in Man (OMIM) database (https:// omim.org/).

\section{Validation of variants and co-segregation analysis}

Sanger sequencing was used to ascertain whether the candidate variants co-segregated with the disease phenotype present in the family. The genomic regions containing these variants were amplified. Polymerase chain reaction (PCR) products were sequenced using an Applied Biosystems (ABI) 3730XL Genetic Analyzer (Applied Biosystems, Foster City, CA, USA) in accordance with the manufacturer's instructions for BigDye ${ }^{\mathrm{TM}}$ Terminator v3.1 Cycle Sequencing Kits. Primers for Sanger sequencing are shown in Table S1.

\section{Tooth structure investigations}

Scanning electron microscopy (SEM) investigation: The primary tooth from the proband (IV:1) was removed due to severe periapical infection. A primary deciduous tooth from an age-matched normal individual was used as the control. After removal of the periodontal membrane and other objects, the tooth samples were immersed in a $10 \%$ formaldehyde solution at $4{ }^{\circ} \mathrm{C}$. The samples were then cross-cut into 2 parts by dental diamond burs and inlaid with epoxy resin. They were then polished with 600, 1,000, $2,000,3,000,5,000$, and 7,000 mesh water sandpaper (Starcke, Melle, Germany) in sequence and ultrasonically cleaned with distilled water. After drying, their surfaces were coated with gold in a vacuum, and the cross-section surfaces were observed using SEM (Inspect F50, FEI Co., Hillsboro, OR, USA).

Energy dispersive spectroscopy (EDS) investigation: the EDS results were analyzed using an SEM (FEI Co.) equipped with an EDS INCA system (Oxford Instruments Analytical, Abingdon, UK). The EDS was conducted at a high accelerating voltage of $20 \mathrm{kV}$. Elemental measurements $(\mathrm{Na}, \mathrm{Mg}, \mathrm{P}, \mathrm{Ca})$ were repeated 3 times in the middle of the dentin, and the $\mathrm{Ca} / \mathrm{P}$ ratio was calculated.

\section{Construction of plasmids}

The coding region of the whole human DSP protein (from aa 1 to aa 382) was cloned into the Hind III/BamHI sites of the pEGFP-N1 vector, as previously described (16), namely DSPpEGFP-N1-WT. The c.53T > G (p. Val18Gly) mutation was introduced into the DSP-pEGFP-N1-WT using a Q5 ${ }^{\circledR}$ SiteDirected Mutagenesis Kit (New England Biolabs, Ipswich, MA, USA), namely DSP-pEGFP-N1-MUT.

\section{Protein localization analysis}

DSPP is not expressed in the HEK293 cells, so we choose this cell line to explore the location of the DSPP mutant. HEK293 cells were cultured in 24-well plates with Dulbecco's modified Eagle's medium (DMEM; Gibco, Grand Island, NY, USA) supplemented with $10 \%$ fetal bovine serum (FBS; Gibco ${ }^{\circledR}$, USA), $100 \mathrm{unit} / \mathrm{mL}$ penicillin, and $100 \mu \mathrm{g} / \mathrm{mL}$ streptomycin. The cells were transfected with DSP-pEGFP-N1-WT or DSP-pEGFP-N1-MUT using Lipofectamine 3000 reagent (Invitrogen, Carlsbad, CA, USA). After $36 \mathrm{~h}$ of transfection, protein localization was detected by immunocytochemistry (ICC). The slides were incubated overnight at $4{ }^{\circ} \mathrm{C}$ with rabbit polyclonal anticalnexin (CANX) antibody (cat: A0692, ABClonal, Wuhan, China) or mouse polyclonal anti-Golgi matrix protein (GM130) antibody (cat: ab169276, Abcam, Cambridge, UK) at a dilution of $1: 600$, followed by incubation with Alexa Fluor 594-conjugated goat anti-rabbit $\operatorname{IgG}(\mathrm{H}+\mathrm{L})$ 
(cat: AS039, ABClonal, Wuhan, China) or Alexa Fluor 594-conjugated goat anti-mouse $\operatorname{IgG}(\mathrm{H}+\mathrm{L})$ (cat:A-11005, Invitrogen, USA) for $2 \mathrm{~h}$. Subsequently, the cells were incubated for $2 \mathrm{~h}$ with 4',6-diamidino-2-phenylindole (DAPI). Cells were imaged using a confocal microscope (Zeiss LSM 800, Jena, Germany).

\section{Isolation and identification of buman dental pulp stem cells (bDPSCs)}

hDPSCs were isolated and cultured from the pulp of primary teeth of the proband (IV:1) and 3 age-matched healthy children, as described in previous studies (17). The cells were stored in liquid nitrogen until use. The hDPSCs were identified by mesenchymal markers using FACSCantoII [BD Bioscience ${ }^{\circledR}$ (Becton, Dickinson, and Co.), Franklin Lakes, NJ, USA]. We used APC-conjugated anti-CD29 antibody, BV510-conjugated anti-CD33 antibody, PE-conjugated anti-CD34 antibody, PerCPCy5.5-conjugated anti-CD44 antibody, FITC-conjugated anti-CD90, and BV421-conjugated anti-CD105 antibody to detect the expression of mesenchymal markers in hDPSCs.

\section{Mineralization assay}

The P3 hDPSCs were inoculated in a $6 \mathrm{~cm}$ dish at the density of $1 \times 10^{5}$ cells per dish. After growing to $80 \%$ of the dish in DMEM, cells were cultured in growth medium supplemented with $50 \mu \mathrm{g} / \mathrm{mL}$ ascorbic acid, $10 \mathrm{mM}$ $\beta$-glycerophosphate, and $100 \mathrm{nM}$ dexamethasone for 7 days. The inoculated cells were washed with phosphate-buffered saline (PBS) 3 times and fixed with 4\% paraformaldehyde for $30 \mathrm{~min}$ at room temperature. Alizarin red $\mathrm{S}$ dye was then added for $10 \mathrm{~min}$, and the cells were washed again with PBS 3 times. The staining intensity was quantified using Image J software (https://imagej.nih.gov/ij/).

\section{$R N A$ extraction and quantitative real-time PCR (qRT-PCR)}

The P3 hDPSCs were cultured in growth medium supplemented with $50 \mu \mathrm{g} / \mathrm{mL}$ ascorbic acid, $10 \mathrm{mM}$ $\beta$-glycerophosphate, and $100 \mathrm{nM}$ dexamethasone for 7 days. Total RNA (100 ng) from each sample was extracted using TRIzol (TransGen ${ }^{\circledR}$, China) and was used for cDNA synthesis using the TransScript Reverse Transcription System (TransGen Biotech ${ }^{\circledR}$, Beijing, China). We then used qRT-PCR to measure the messenger RNA (mRNA) levels of DSPP, ALP, and RUNX2 using ChamQTM Universal SYBR Qpcr Master Mix (Vazyme ${ }^{\circledR}$, Nanjing, China). Glyceraldehyde 3-phosphate dehydrogenase (GAPDH) was used as an internal control for standardization. Primers for each gene are shown in Table S1. Relative gene expression levels were calculated using the $2^{-\Delta \Delta C T}$ method.

\section{Statistical analysis}

All experiments were repeated 3 times. All data were expressed as the mean \pm SD. Student's $t$-test was used to determine the statistical significance. Statistical significance was considered when $\mathrm{P}<0.05$.

\section{Results}

\section{Clinical and radiographic features}

The 4-generation Chinese family with DGI included 4 affected family members and 6 unaffected members. There were 2 affected members and 1 healthy member referred for clinical assessment (Figure 1A).

The primary teeth of the 7-year-old proband (IV:1) had a typical yellow-brown color and showed severe attrition. The newly erupted first permanent molars and permanent incisors exhibited hypoplastic enamel defects. The enamel defects of the newly erupted teeth were near the incisal edge, and the enamel defects of the molars were on the occlusal surface of the crown (Figure 1B). Notably, even though the mandibular right first molar was newly erupted and seemingly intact, a periapical shadow and fistula appeared (Figure 1C,1D). Radiographic examination revealed hypoplastic enamel defects on unerupted incisors and canines near the incisal edge, suggesting that enamel formation was affected. A certain amount of pulpal obliteration had occurred in newly erupted teeth, but the pulp chambers were still accessible (Figure 1E,1F).

The father of the 7-year-old (III: 1) showed severe vertical dimension loss. Upon intraoral examination, the teeth were typically yellow-brown in color and translucent, with severe tooth attrition to the level of the gingiva (Figure 1G,1H). The oral panoramic radiograph showed that the pulp cavities were totally obliterated and the roots were short (Figure 1I). The CBCT images revealed pathological attrition of the left mandibular head and visible rotation backwards of the mandibular head of the temporalmandibular joint (TMJ) due to vertical dimensional loss (Figure 17). 
A

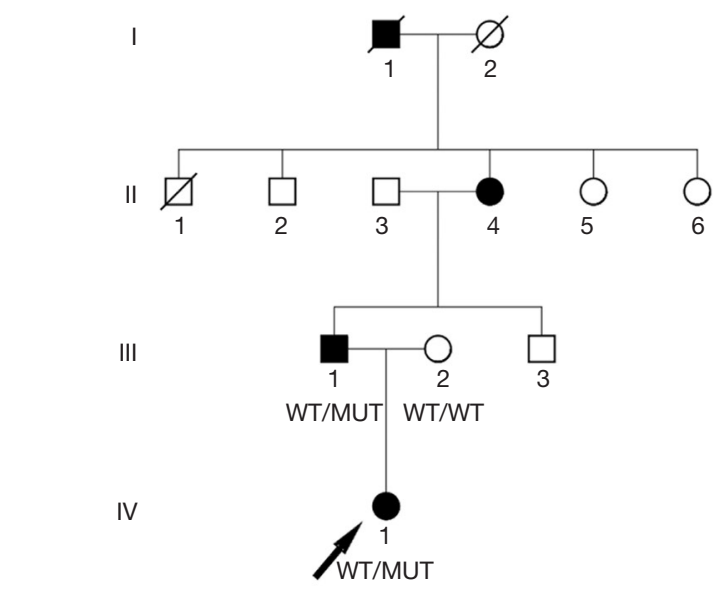

B
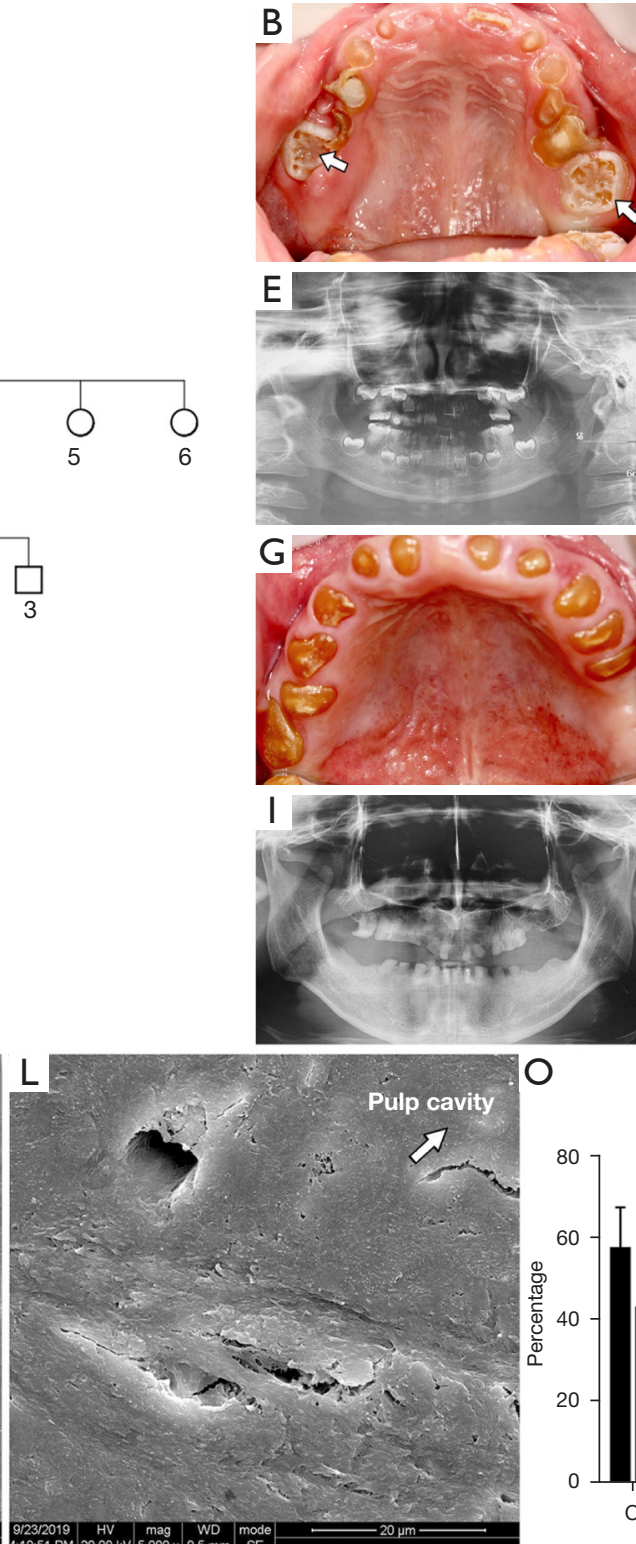

0
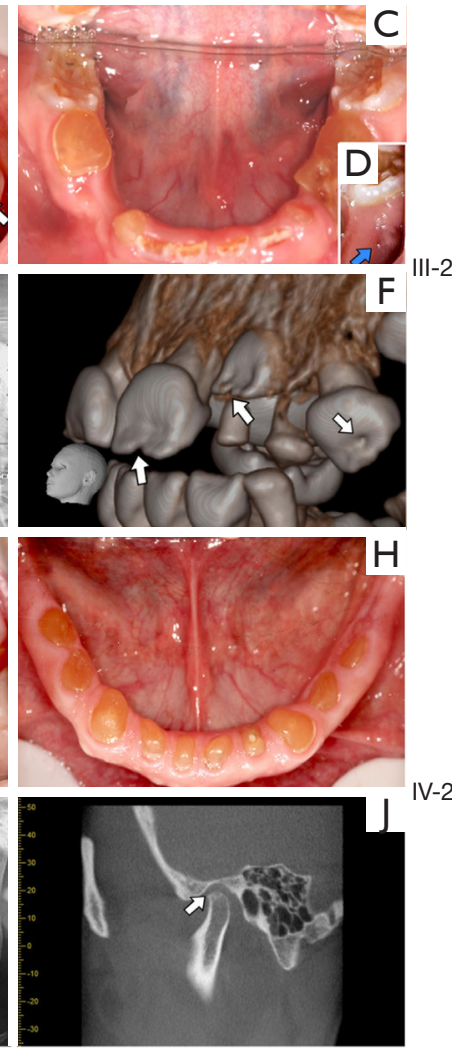

IV-2
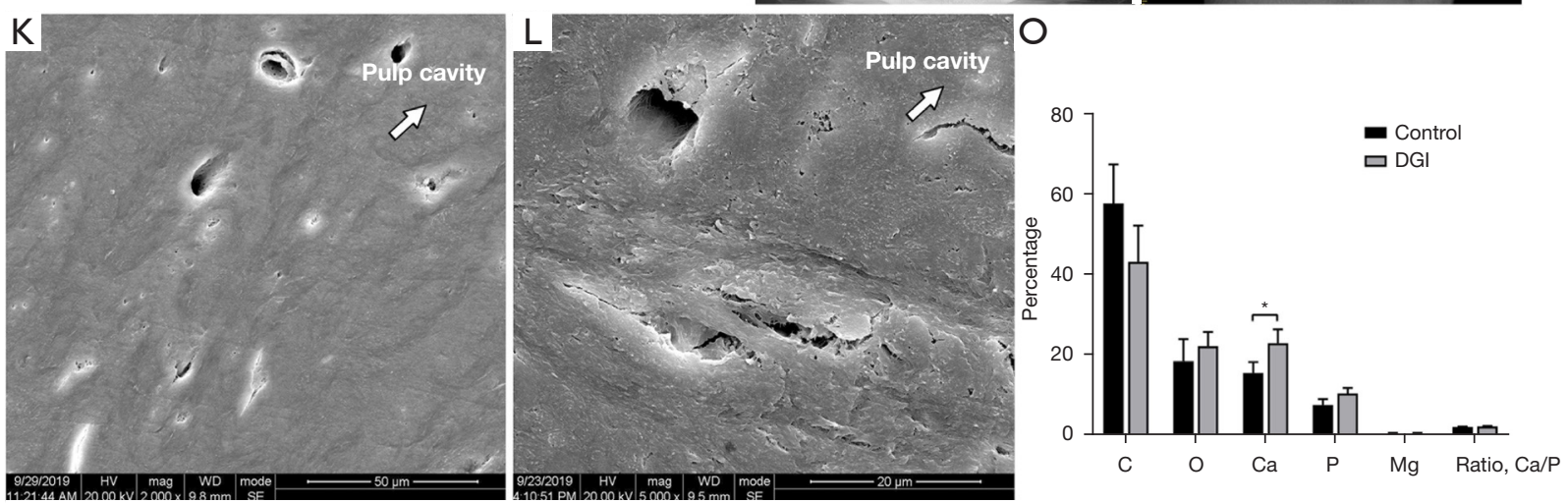

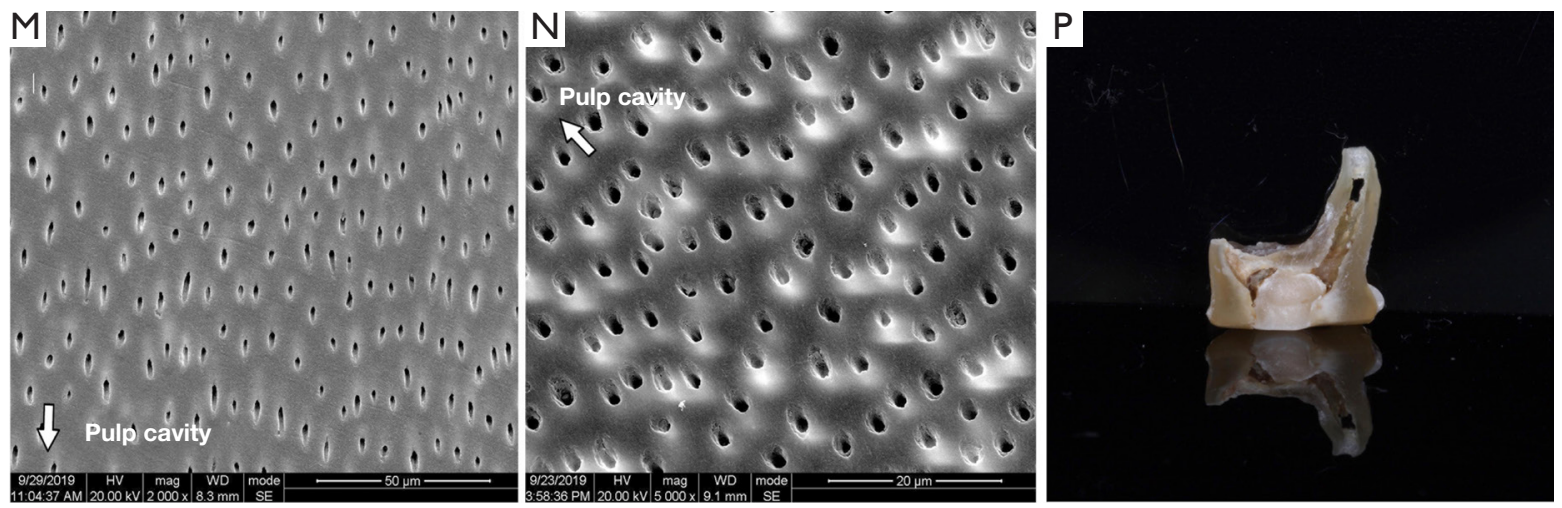

Figure 1 Pedigrees of the DGI family, clinical examinations, and tooth structure investigations. (A) Pedigrees of the DGI family. (B-F) The clinical and radiographic findings for IV.1. (B) Facial and intraoral examinations showing the enamel defect on newly erupted teeth (white arrows). (C,D) Soon after the eruption of the mandibular right first molar, a fistula appeared (blue arrow). (E) The oral panoramic radiographs show that the teeth pulp cavities of unerupted teeth were available, while the cavities of the erupted teeth were partially obliterated. (F) The CBCT image of the unerupted teeth shows hypoplastic enamel defects (white arrows). (G-J) The clinical and radiographic finding for of III.1. (G,H) Teeth are yellow-brownish colored and show severe attrition to the gingiva margin. (I) The oral panoramic radiographs show that the teeth pulp cavities are totally obliterated, and the roots are short and round. (J) The CBCT image shows that the 2 mandibular heads are asymmetric; the left mandibular head shows attrition and is rotated forward (white arrow). (K) SEM image of the DGI tooth (2,000×). (L) SEM image of the DGI tooth $(5,000 \times)$. (M) SEM image of the control (2,000×). (N) SEM image of the control $(5,000 \times)$. (White arrows indicate the direction of pulp cavity). (O) EDS results of the DGI tooth and the control, *, P<0.05. (P) Image of the DGI tooth. DGI, dentinogenesis imperfecta; CBCT, cone-beam computed tomography; SEM, scanning electron microscopy; EDS, energy dispersive spectroscopy.

\section{Investigation of tooth structures}

The SEM showed that the dentinal tubules of patient teeth were loosely arranged, highly irregular in size and shape, and reduced in number (Figure $1 K, 1 L$ ), while the normal dentinal tubules were highly regular in shape and size (Figure $1 M, 1 N$ ). The EDS results showed that the calcium content of the DGI dentin was significantly higher than that of the normal dentin $(23.010 \pm 3.483$ vs. $15.527 \pm 2.789$, $\mathrm{P}=0.044)$. There were no significant differences in other elements of the DGI teeth and normal teeth (Figure 10, Table S2). The teeth had a typical yellow brownish color, the root canal was accessible, and there was seldom any existing enamel (Figure 1P).

\section{Genetic findings}

A novel heterozygous c.53T > G (p. Val18Gly) variant on exon 3 of the DSPP gene was identified in the proband (IV:1) by WES. The 2 affected individuals were heterozygous for the variant, and the variant was not found in the unaffected family member. The c.53T > G (p. Val18Gly) variant in DSPP was completely co-segregated with the disease phenotype in the family (Figure $2 A$ ). This variant was absent in the 1000 Genomes Project, the ESP6500 database, GnomAD, and an in-house database generated from 1,092 samples of WES. The variant occurs in an evolutionarily conserved amino acid through the protein sequence alignment of orthologs from 9 different species (Figure 2B). The c.53T > G (p. Val18Gly) variant was predicted by Sorting Intolerant from Tolerant (SIFT) and Polyphen2 to be damaging (Table S3). The variant was revealed to cause valine to become glycine at position 18 , which is very flexible and may destroy the rigidity of the protein at this position. The size and hydrophobicity of mutant residues were found to be different from those of the wild-type DSP, which also changes the original characteristics of the DSP protein (Figure 2C). The novel mutation was classified as a pathogenic variant according to the ACMG interpretation guidelines (15).

\section{Effect of DSPP gene variants on protein localization}

To determine the location of the DSPP mutants, the anticalnexin (CANX) antibody and anti-Golgi matrix protein 
A

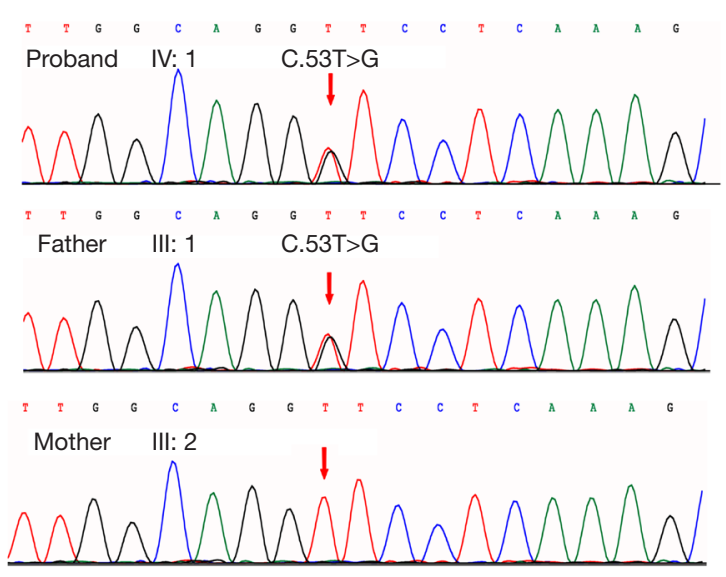

B

\begin{tabular}{|c|c|c|}
\hline $\begin{array}{l}\text { imp } \\
\text { esus } \\
\text { ouse } \\
\text { t } \\
\text { g } \\
\text { nobo } \\
\text { rilla }\end{array}$ & 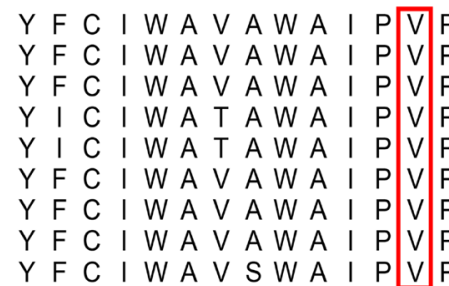 & \\
\hline
\end{tabular}

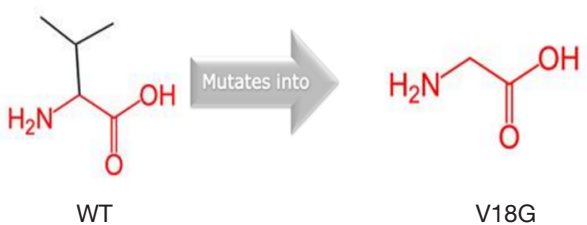

Figure 2 DSPP (NM_014208) variations were identified by Sanger sequencing in participants. (A) Sanger sequencing of the c.53T > G (p. Val18Gly) variant in DSPP. (B) Evolutionary conservation of the DSPP protein. Multiple amino acid sequence alignment of DSPP indicated conservation of valine (shown in red) at position 18 in different species. (C) The size and hydrophobicity of mutant residues are different from those of the wild type, which also changes the original characteristics of the DSP protein. WT, wild-type; DSPP, dentin sialophosphoprotein; DSP, dentin sialoprotein.

(GM130) antibodies were used to stain HEK-293 cells transfected with DSP-pEGFP-N1-WT or DSP-pEGFPN1-MUT. Wild-type DSP was found predominantly in the Golgi apparatus. The p. Val18Gly mutant DSP was found predominantly in the endoplasmic reticulum (ER), even though a portion was localized in the Golgi apparatus (Figure $3 A-3 D$ ), which is consistent with previous reports $(16,18)$.

\section{Phenotypic and functional characteristics of DGI bDPSCs}

After seeding in culture dishes, both control cells and patient cells exhibited a characteristic fibroblast-like morphology of hDPSCs (Figure S1A,S1B). The cells were positive for CD29, CD44, CD90, and CD105 and negative for CD33 and CD34, indicating mesenchymal stem cell markers (Figure S1C,S1D). The mineralization assay showed that mineralized calcium deposition in the DGI cells was significantly lower than that in the control cells $(\mathrm{P}=0.012)$ (Figure $4 A, 4 B$, Table S4).

The qRT-PCR analysis showed that the expression levels of DSPP $(\mathrm{P}=0.022)$ and RUNX2 $(\mathrm{P}=0.007) \mathrm{mRNA}$ in DGI cells were significantly lower than those in the control. The ALP mRNA levels were similar between 2 groups $(\mathrm{P}=0.866)$ (Figure 4C-4E, Table S5).

\section{Discussion}

The dental disorder, DGI, Shields type-II is a rare hereditary dentin defect. Thus, recruitment of large cohort of cases is difficult, making it difficult to study the clinical features of patients to better understand the genotypephenotype relationship. In our study, the teeth of patients presented with typical clinical manifestations including yellow-brownish appearance and severe attrition. The roots were narrower than those of normal teeth, and pulp chambers and root canals were smaller than those in normal teeth, as well as being partially obliterated. The enamel was more prone to fracture, and the exposed dentin was severely and rapidly worn. So, dental examination should be performed soon after teeth erupt, and dental managements should accompany the patients for life. Performed metal crowns should be applied on the primary molars to maintain chewing function. The primary anterior teeth are recommended to restored with resin crowns for beauty considerations and to maintain vertical height (19). In this study, the patients also showed primary hypoplastic enamel defects. In another Korean family with DGI, Shields type II, hypoplastic enamel defects were also found in teeth, which is similar to the phenotype in this study (20). Furthermore, enamel defects have been observed in 2 Caucasian families 

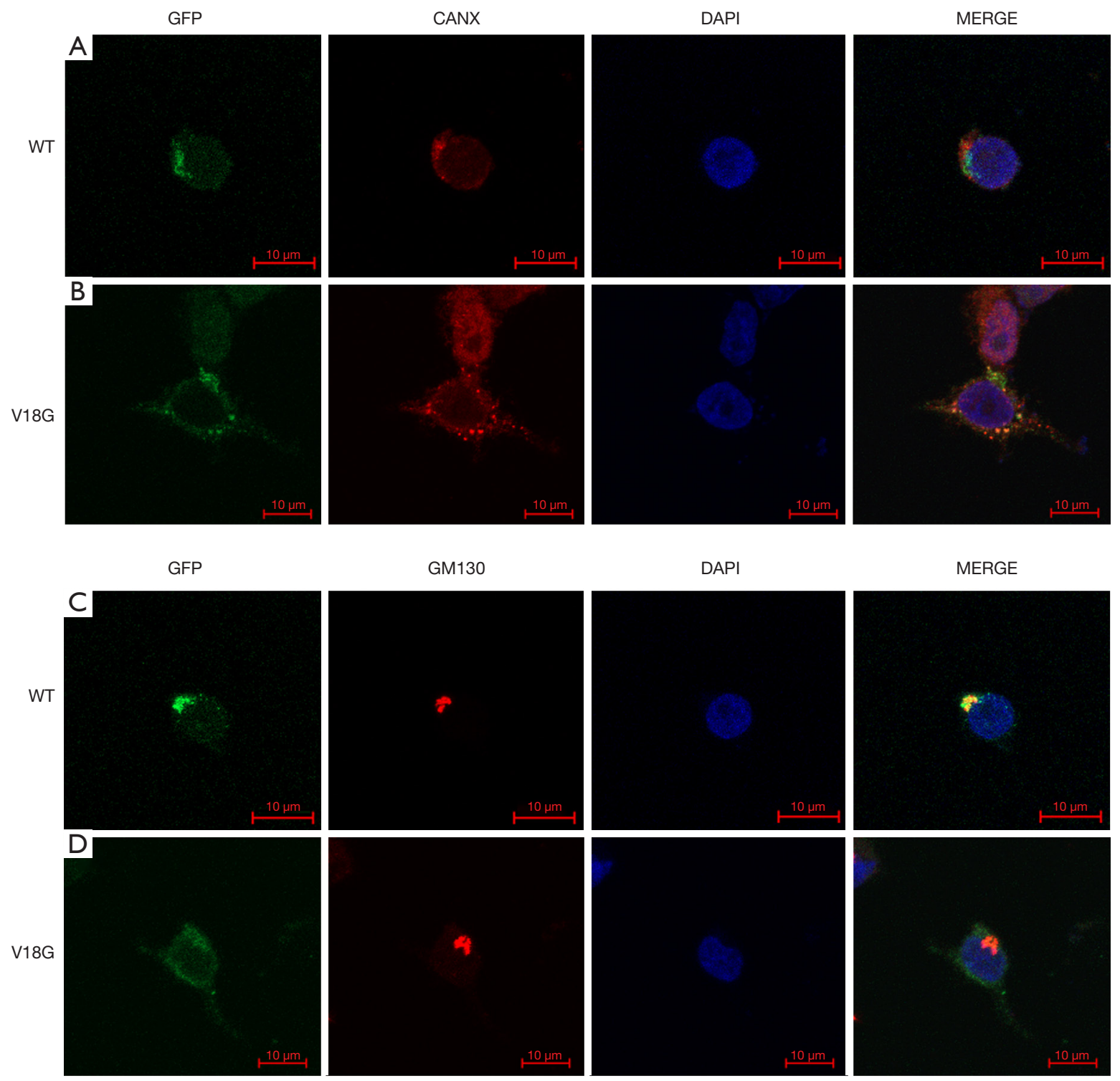

Figure 3 Protein localization of wild-type and mutant of DSPP gene. Confocal microscopy shows intracellular localization of the GFPtagged WT and mutant (p. Val18Gly) DSP in HEK293T cells. Anti-CANX antibody was used for ER staining, and a GM130 antibody was used for Golgi staining. Nuclei were stained with DAPI. (A,C) Wild-type DSP was predominantly found in the Golgi apparatus. (B,D) The p. Val18Gly mutant DSP was found predominantly in the ER, even though a portion was localized in the Golgi apparatus. GFP, green fluorescent protein; WT, wild-type; DSPP, dentin sialophosphoprotein; DSP, dentin sialoprotein; CANX, anti-calnexin; GM130, anti-Golgi matrix protein; ER, endoplasmic reticulum; DAPI, 4',6-diamidino-2-phenylindole. 


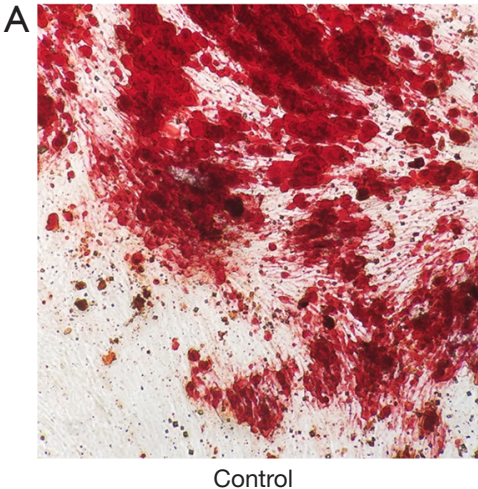

C

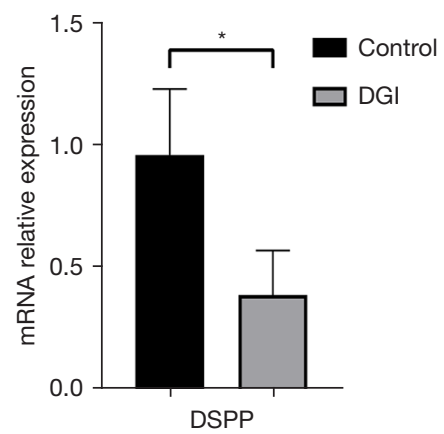

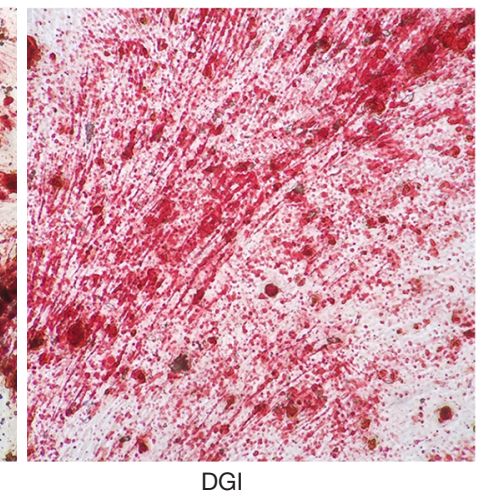

D

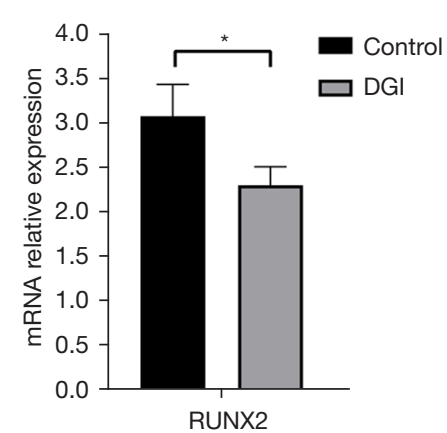

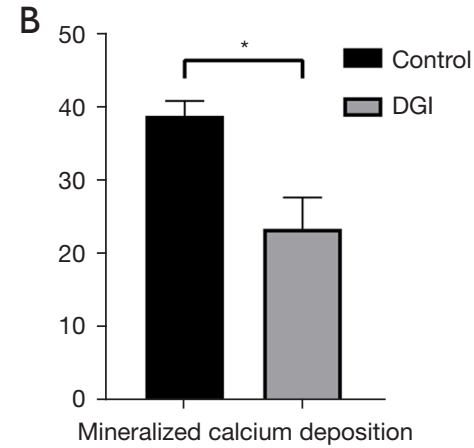

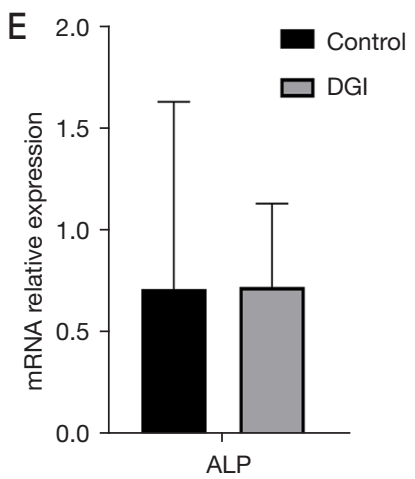

Figure 4 Phenotypic and functional characteristics of hDPSCs from DGI patient. (A) Accumulation of calcium stained with alizarin red staining after growth of control cells and DGI cells $(\times 10)$. (B) The mineralization ability of DGI cells was significantly lower than control cells, *, $\mathrm{P}<0.05$. (C) The expression level of DSPP in DGI cells was significantly lower than in control cells, ${ }^{*}, \mathrm{P}<0.05$. (D) The expression level of RUNX2 in DGI cells was significantly lower than in control cells, ${ }^{*}, \mathrm{P}<0.05$. (E) The expression level of ALP in DGI and control cells showed no significant difference. hDPSCs, human dental pulp stem cells; DGI, dentinogenesis imperfecta; DSP, dentin sialoprotein; RUNX2, runt-related transcription factor 2; ALP, alkaline phosphatase.

with classic DGI, Shields type II phenotype, which had the same mutation of c. $143+1 \mathrm{G}>\mathrm{A}$ in the exon 3 flanker of DSPP (21). The reason may be that DSPP is not only expressed by odontoblasts (22-24), but also transiently expressed by ameloblasts (25). Verdelis et al. (26) found that $D S P P$ is important for normal mineralization of both dentin and enamel. In comparison with control mice, $D S P P^{-/-}$ mice showed reduced thickness of the outer enamel layer, while the total enamel thickness remained unchanged. However, the role of DSPP in amelogenesis remains largely unclear and further studies are needed to investigate the associations between DSPP and these clinical phenotypic heterogeneities.

A novel heterozygous c.53T > G (p. Val18Gly) variant was identified in DSPP. The novel mutation was classified as a pathogenic variant according to the interpretation guidelines of ACMG (15). This mutation leads to the replacement of valine with glycine at codon 18 . Several studies have shown that mutations in DSPP may cause distinct phenotypes. For example, c.53T > A (Val18Asp) is associated with DGI, Shields type II $(20,27,28)$. Substitutions of the c.52G > T (Val18Phe) cause DGI, Shields type II and DGI, Shields type I with progressive sensorineural high-frequency hearing loss. No enamel defect was observed in patients with the mutation c.52G $>$ T (Val18Phe) $(10,29)$, and in patients with the mutation c. $53 \mathrm{~T}>\mathrm{A}$ (Val18Asp) in Japanese and Korean families with DGI, Shields type II $(27,28)$. These data reveal the complexity of the relationship between genotype and clinical phenotype.

The SEM results showed that the DGI teeth had reduced and irregular dentinal tubes as previously reported (30,31). The EDS results showed that the DGI dentin had elevated Ca contents, which is consistent with previous 
studies in DGI, Shields type II patients (32). During tooth formation, hDPSCs differentiate into odontoblasts and participate in the dentin formation process $(33,34)$. The DSPP gene plays an important role in cell differentiation and tooth morphogenesis (35-37). Both DSP and DPP are considered to play a role in dentin mineralization; however, differing from the established role of DPP during dentin mineralization, the role of DSP is still not clear (38). Suzuki's research (8) has shown that DSP may partially rescue the dentin volume but not the dentin mineral density, indicated DSP regulating the initiation of dentin mineralization. Gibson et al. (39) showed that dentin was more poorly mineralized and remarkably disorganized in DSP-PP null mice than in the DSP-PP KO mice, indicating that DSP may inhibit dentin mineralization or serve as an antagonist of the accelerating action of DPP. In this study, mineralized calcium deposition in the hDPSCs of the DGI patient was significantly lower than in the control cells, indicating impaired mineralization ability of the DGI cells. The EDS results showed that the calcium content of the DGI dentin was significantly higher than in normal dentin. The reason may be that besides the initial mineralization process, differences in set of teeth, age, and dentin ultrastructure may cause the difference in mineral composition (40). In their study, Park et al. (41), reported that the DGI maxillary first molars of 9-year-old girl showed lower $\mathrm{Mg}$ content but no difference in $\mathrm{Ca}$ content and $\mathrm{Ca} / \mathrm{P}$ ratio, which is consistent with previous study (42) of DGI primary teeth. Several other studies had different results, in Kerebel et al.'s (43) research, lower content of $\mathrm{Ca}, \mathrm{P}$, and $\mathrm{Mg}$ was found in the DGI dentin, while in Li et al.'s study (32), a higher content of Ca was found in the dentin of a permanent DGI tooth. In Intarak et al.'s (40) study of osteogenesis imperfecta patients, whose teeth showed typical DGI manifestation, the Ca content of the younger patient was comparable with the control while the older patient's teeth showed significantly lower $\mathrm{Ca}$ content, indicating that the calcium and phosphorus contents may be related to age.

DPSCs may differentiate into odontoblasts and regenerate a dentin-pulp-like complex to form restorative dentin. The differentiation process of DPSCs induced to odontoblasts in vitro is similar to the differentiation process of odontoblasts in vivo, which give us a chance to observe the physiological activities of DGI mutant cells. The expression level of DSPP and RUNX2 were significantly lower in DGI hDPSCs than in those of the control. The $D S P P$ gene plays an important role in the process of tooth development, and the spatiotemporal expression of DSPP is finely manipulated during different stages of dental formation. The RUNX2 gene is a critical transcriptional regulator during early tooth formation, contributing to the spatiotemporal expression of DSPP during dentinogenesis $(44,45)$. Reduced expressions of DSPP and RUNX2 led to impaired ability of the DGI hDPSCs, a result consistent with the findings of previous studies $(5,31)$. The expression of $A L P$ showed no difference between the 2 groups. Mineralization is controlled by DSPP, RUNX2, ALP, and other small integrin-binding ligand $\mathrm{N}$-linked glycoproteins (SIBLINGs), such as dentine morphogenetic protein 1 (DMP1), bone sialoprotein (BSP), and osteopontin (OPN) $(46,47)$. Future studies should aim to explain this complex mineralization process.

The ICC results showed that this novel DSPP mutation caused retention of the DSP protein in the ER, while the WT DSP protein was secreted to the Golgi apparatus. This novel mutation c.53T > G (p. Val18Gly) is located on the third isoleucine-proline-valine (IPV) motif. Previous studies $(5,18)$ have shown that mutations in the IPV of DSPP may cause DSPP to be retained in the ER, which may lead to the dominant negative effects.

In summary, we identified a novel heterozygous c.53T > G (p. Val18Gly) DSPP variant in a family with DGI with rare hypoplastic enamel defects. Functional analyses revealed that this novel variant disturbs dentinal characteristics and pulp cell behavior by impairing DSPP function. These results extended our knowledge of the genetic mutation spectrum of DSPP. Further research is needed to fill the gap between specific DSPP mutation sites and certain clinical features.

\section{Acknowledgments}

We thank all the participants in the family.

Funding: This work was supported by The Research Foundation of Science and Technology Bureau of Chengdu (2015-HM02-00094-SF, to JY) and the Applied Foundation in Science and Technology Office of Sichuan (2021YFG0230, to QD).

\section{Footnote}

Reporting Checklist: The authors have completed the MDAR reporting checklist. Available at https://dx.doi. org/10.21037/atm-21-5369

Data Sharing Statement: Available at https://dx.doi. 
org/10.21037/atm-21-5369

Conflicts of Interest: All authors have completed the ICMJE uniform disclosure form (available at https://dx.doi. org/10.21037/atm-21-5369). The authors have no conflicts of interest to declare.

Ethical Statement: The authors are accountable for all aspects of the work in ensuring that questions related to the accuracy or integrity of any part of the work are appropriately investigated and resolved. This study was approved by the Medical Ethics Review Committee of Sichuan Provincial People's Hospital [No. 2020(02)]. All procedures performed in the study involving human participants were in accordance with the ethical standards of the Medical Ethics Review Committee of Sichuan Provincial People's Hospital and the Declaration of Helsinki (as revised in 2013). Informed consent was provided by all individual participants included in the study.

Open Access Statement: This is an Open Access article distributed in accordance with the Creative Commons Attribution-NonCommercial-NoDerivs 4.0 International License (CC BY-NC-ND 4.0), which permits the noncommercial replication and distribution of the article with the strict proviso that no changes or edits are made and the original work is properly cited (including links to both the formal publication through the relevant DOI and the license). See: https://creativecommons.org/licenses/by-nc-nd/4.0/.

\section{References}

1. Nieminen P, Papagiannoulis-Lascarides L, Waltimo-Siren $\mathrm{J}$, et al. Frameshift mutations in dentin phosphoprotein and dependence of dentin disease phenotype on mutation location. J Bone Miner Res 2011;26:873-80.

2. Malmgren B, Andersson K, Lindahl K, et al. Tooth agenesis in osteogenesis imperfecta related to mutations in the collagen type I genes. Oral Dis 2017;23:42-9.

3. Shields ED, Bixler D, el-Kafrawy AM. A proposed classification for heritable human dentine defects with a description of a new entity. Arch Oral Biol 1973;18:543-53.

4. Witkop CJ Jr. Hereditary defects of dentin. Dent Clin North Am 1975;19:25-45.

5. Liang T, Zhang H, Xu Q, et al. Mutant Dentin Sialophosphoprotein Causes Dentinogenesis Imperfecta. J Dent Res 2019;98:912-9.

6. Garrocho-Rangel A, Dávila-Zapata I, Martínez-Rider R, et al. Dentinogenesis Imperfecta Type II in Children: A Scoping Review. J Clin Pediatr Dent 2019;43:147-54.

7. Fan F, Li N, Huang S, et al. A multidisciplinary approach to the functional and esthetic rehabilitation of dentinogenesis imperfecta type II: A clinical report. J Prosthet Dent 2019;122:95-103.

8. Suzuki S, Sreenath T, Haruyama N, et al. Dentin sialoprotein and dentin phosphoprotein have distinct roles in dentin mineralization. Matrix Biol 2009;28:221-9.

9. Ogbureke KU, Fisher LW. SIBLING expression patterns in duct epithelia reflect the degree of metabolic activity. J Histochem Cytochem 2007;55:403-9.

10. Xiao $\mathrm{S}, \mathrm{Yu} \mathrm{C}, \mathrm{Chou} \mathrm{X}$, et al. Dentinogenesis imperfecta 1 with or without progressive hearing loss is associated with distinct mutations in DSPP. Nat Genet 2001;27:201-4.

11. Zhang X, Zhao J, Li C, et al. DSPP mutation in dentinogenesis imperfecta Shields type II. Nat Genet 2001;27:151-2.

12. Rajpar MH, Koch MJ, Davies RM, et al. Mutation of the signal peptide region of the bicistronic gene DSPP affects translocation to the endoplasmic reticulum and results in defective dentine biomineralization. Hum Mol Genet 2002;11:2559-65.

13. Song YL, Wang CN, Fan MW, et al. Dentin phosphoprotein frameshift mutations in hereditary dentin disorders and their variation patterns in normal human population. J Med Genet 2008;45:457-64.

14. Liu S, Xie L, Yue J, et al. Whole-exome sequencing identifies a novel homozygous frameshift mutation in the PROM1 gene as a causative mutation in two patients with sporadic retinitis pigmentosa. Int $\mathrm{J}$ Mol Med 2016;37:1528-34.

15. Richards S, Aziz N, Bale S, et al. Standards and guidelines for the interpretation of sequence variants: a joint consensus recommendation of the American College of Medical Genetics and Genomics and the Association for Molecular Pathology. Genet Med 2015;17:405-24.

16. Lee SK, Lee KE, Song SJ, et al. A DSPP mutation causing dentinogenesis imperfecta and characterization of the mutational effect. Biomed Res Int 2013;2013:948181.

17. Gronthos S, Mankani M, Brahim J, et al. Postnatal human dental pulp stem cells (DPSCs) in vitro and in vivo. Proc Natl Acad Sci U S A 2000;97:13625-30.

18. von Marschall Z, Mok S, Phillips MD, et al. Rough endoplasmic reticulum trafficking errors by different classes of mutant dentin sialophosphoprotein (DSPP) cause dominant negative effects in both dentinogenesis imperfecta and dentin dysplasia by entrapping normal 
DSPP. J Bone Miner Res 2012;27:1309-21.

19. Soliman S, Meyer-Marcotty P, Hann B, et al. Treatment of an adolescent patient with dentinogenesis imperfecta using indirect composite restorations - A case report and literature review. J Adhes Dent 2018;20:345-54.

20. Lee SK, Lee KE, Hwang YH, et al. Identification of the DSPP mutation in a new kindred and phenotype-genotype correlation. Oral Dis 2011;17:314-9.

21. Wang SK, Chan HC, Rajderkar S, et al. Enamel malformations associated with a defined dentin sialophosphoprotein mutation in two families. Eur J Oral Sci 2011;119 Suppl 1:158-67.

22. Kim JW, Simmer JP. Hereditary dentin defects. J Dent Res 2007;86:392-9.

23. Baba O, Qin C, Brunn JC, et al. Detection of dentin sialoprotein in rat periodontium. Eur J Oral Sci 2004;112:163-70.

24. Liu Q, Gibson MP, Sun H, et al. Dentin sialophosphoprotein (DSPP) plays an essential role in the postnatal development and maintenance of mouse mandibular condylar cartilage. J Histochem Cytochem 2013;61:749-58. Erratum in J Histochem Cytochem 2014;62:759.

25. Bègue-Kirn C, Krebsbach PH, Bartlett JD, et al. Dentin sialoprotein, dentin phosphoprotein, enamelysin and ameloblastin: tooth-specific molecules that are distinctively expressed during murine dental differentiation. Eur J Oral Sci 1998;106:963-70.

26. Verdelis K, Szabo-Rogers HL, Xu Y, et al. Accelerated enamel mineralization in Dspp mutant mice. Matrix Biol 2016;52-54:246-59.

27. Lee SK, Lee KE, Jeon D, et al. A novel mutation in the DSPP gene associated with dentinogenesis imperfecta type II. J Dent Res 2009;88:51-5.

28. Kida M, Tsutsumi T, Shindoh M, et al. De novo mutation in the DSPP gene associated with dentinogenesis imperfecta type II in a Japanese family. Eur J Oral Sci 2009;117:691-4.

29. Kim JW, Hu JC, Lee JI, et al. Mutational hot spot in the DSPP gene causing dentinogenesis imperfecta type II. Hum Genet 2005;116:186-91.

30. Wieczorek A, Loster J. Dentinogenesis imperfecta type II: ultrastructure of teeth in sagittal sections. Folia Histochem Cytobiol 2013;51:244-7.

31. Porntaveetus T, Osathanon T, Nowwarote N, et al. Dental properties, ultrastructure, and pulp cells associated with a novel DSPP mutation. Oral Dis 2018;24:619-27.

32. Li F, Liu Y, Liu HC, et al. Genetic variants analysis and histological observation of teeth in a patient with hereditary opalescent dentin. Beijing Da Xue Xue Bao Yi Xue Ban 2018;50:666-71.

33. Gu S, Liang J, Wang J, et al. Histone acetylation regulates osteodifferentiation of human dental pulp stem cells via DSPP Front Biosci (Landmark Ed) 2013;18:1072-9.

34. Kawashima N, Okiji T. Odontoblasts: Specialized hardtissue-forming cells in the dentin-pulp complex. Congenit Anom (Kyoto) 2016;56:144-53.

35. Bruderer M, Richards RG, Alini M, et al. Role and regulation of RUNX2 in osteogenesis. Eur Cell Mater 2014;28:269-86.

36. Kim HJ, Kim WJ, Ryoo HM. Post-Translational Regulations of Transcriptional Activity of RUNX2. Mol Cells 2020;43:160-7.

37. Li Q, Yi B, Feng Z, et al. FAM20C could be targeted by TET1 to promote odontoblastic differentiation potential of human dental pulp cells. Cell Prolif 2018;51:e12426.

38. Ritchie $H$. The functional significance of dentin sialoprotein-phosphophoryn and dentin sialoprotein. Int J Oral Sci 2018;10:31.

39. Gibson MP, Liu Q, Zhu Q, et al. Role of the NH2 -terminal fragment of dentin sialophosphoprotein in dentinogenesis. Eur J Oral Sci 2013;121:76-85.

40. Intarak N, Budsamongkol T, Theerapanon T, et al. Tooth ultrastructure of a novel COL1A2 mutation expanding its genotypic and phenotypic spectra. Oral Dis 2021;27:1257-67.

41. Park H, Hyun HK, Woo KM, et al. Physicochemical properties of dentinogenesis imperfecta with a known DSPP mutation. Arch Oral Biol 2020;117:104815.

42. Sabel N, Norén JG, Robertson A, et al. X-ray microanalysis of dentine in primary teeth diagnosed Dentinogenesis Imperfecta type II. Eur Arch Paediatr Dent 2020;21:527-35.

43. Kerebel B, Daculsi G, Menanteau J, et al. The inorganic phase in dentinogenesis imperfecta. J Dent Res 1981;60:1655-60.

44. Chen S, Rani S, Wu Y, et al. Differential regulation of dentin sialophosphoprotein expression by Runx2 during odontoblast cytodifferentiation. J Biol Chem 2005;280:29717-27.

45. Aberg T, Cavender A, Gaikwad JS, et al. Phenotypic changes in dentition of Runx2 homozygote-null mutant mice. J Histochem Cytochem 2004;52:131-9. Erratum in J Histochem Cytochem 2004;52:841.

46. Foster BL, Tompkins KA, Rutherford RB, et al. Phosphate: known and potential roles during development 
and regeneration of teeth and supporting structures. Birth Defects Res C Embryo Today 2008;84:281-314.

47. Rodrigues TL, Foster BL, Silverio KG, et al.

Hypophosphatasia-associated deficiencies in mineralization and gene expression in cultured dental pulp cells obtained from human teeth. J Endod 2012;38:907-12.

(English Language Editor: J. Jones)

Cite this article as: Du Q, Cao L, Liu Y, Pang C, Wu S, Zheng L, Jiang W, Na X, Yu J, Wang S, Zhu X, Yang J. Phenotype and molecular characterizations of a family with dentinogenesis imperfecta shields type II with a novel DSPP mutation. Ann Transl Med 2021;9(22):1672. doi: 10.21037/atm21-5369 


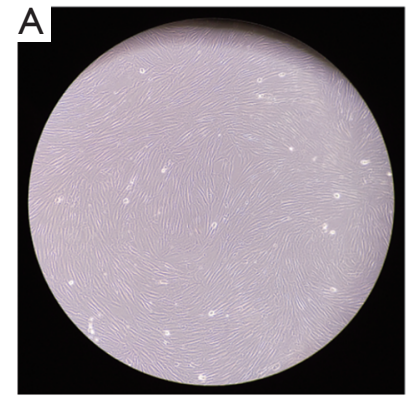

Control cells

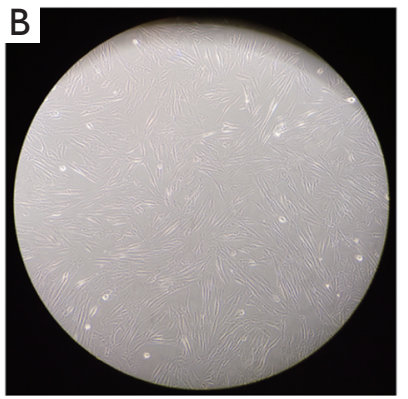

DGI cells
C

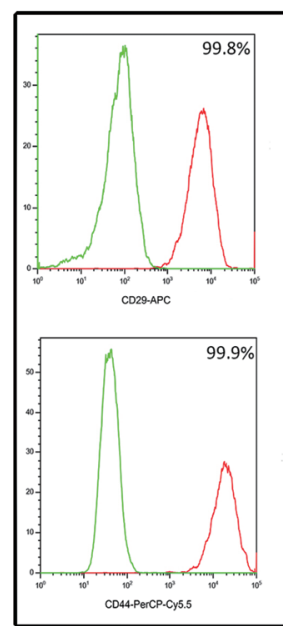

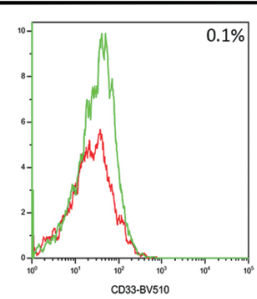

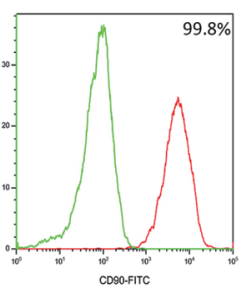

Control
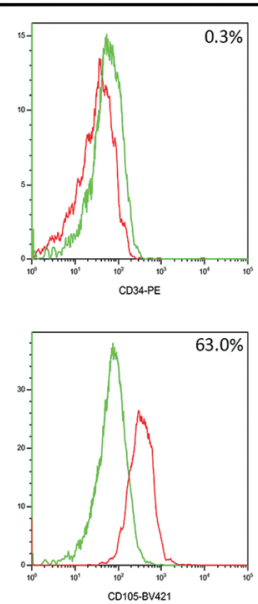
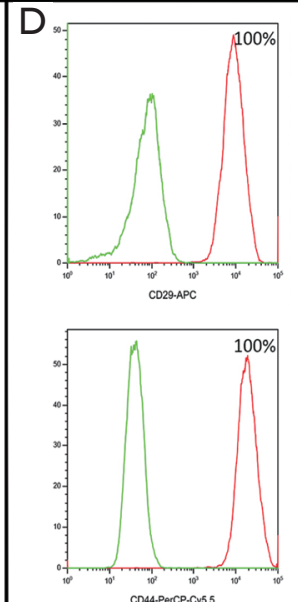

CD44-PerCP-CO5.5
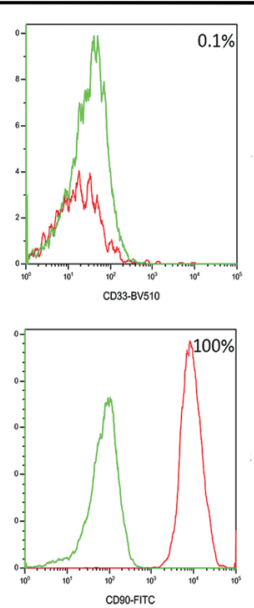

DGI
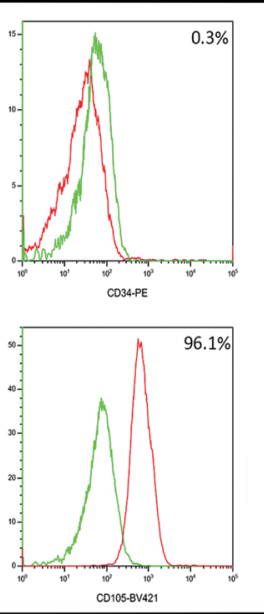

CD105-BV421

Figure S1 Isolation and Identification of hDPSCs. (A) The morphology of the control cells culture $(\times 100)$. (B) The morphology of the patient' cells culture $(\times 100)$. (C) Flow cytometric analysis of control cells. Control cells are positive for CD29, CD44, CD90, and CD105 and negative for CD33 and CD34. (D) Flow cytometric analysis of patient' cells. DGI cells are positive for CD29, CD44, CD90, CD105 and negative for CD33 and CD34. hDPSCs, human dental pulp stem cells; DGI, dentinogenesis imperfecta.

Table S1 Primers used for DSPP gene verification and qPCR

\begin{tabular}{lll}
\hline Amplicon & Primers sequence (5' to 3') & Amplicon size (bp) \\
\hline DSPP, c.53T>G & F-GGGCTGATCTAACACGTCCA & 500 \\
RSPP-qPCR & R-CCACTGGCATTTAACTCATCCT & 168 \\
R-ACACCCAGAAGCTCAACCAT & \\
R-TACCTTCGTTGCCTTCCCA & 169 \\
RUNX2 & F-TAAGGACATCGCCTACCAGCTC & \\
& R-TCTTCCAGGTGTCAACGAGGT & 183 \\
GAPDH & F- CTGTGGTTACTGTCATGGCG & \\
& R-AGGTAGCTACTTGGGGAGGA & 136 \\
& CTITGGTATCGTGGAAGGACTC & \\
\hline
\end{tabular}


Table S2 The element content in DGI teeth and control

\begin{tabular}{lcccccc}
\hline Variable & $\mathrm{C} / \%$ & $\mathrm{O} / \%$ & $\mathrm{Mg} / \%$ & $\mathrm{Ca} / \%$ & $\mathrm{P} / \%$ & $\mathrm{Ratio}(\mathrm{Ca} / \mathrm{P})$ \\
\hline DGl & $43.40 \pm 9.04$ & $22.29 \pm 3.49$ & $0.43 \pm 0.12$ & $23.01 \pm 3.48$ & $10.45 \pm 1.37$ & $2.20 \pm 0.09$ \\
Control & $58.00 \pm 9.76$ & $18.57 \pm 5.48$ & $0.37 \pm 0.20$ & $15.52 \pm 2.79$ & $7.52 \pm 1.48$ & $2.07 \pm 0.08$ \\
P value & 0.130 & 0.377 & 0.672 & 0.044 & 0.066 & 0.140 \\
\hline
\end{tabular}

DGI, dentinogenesis imperfecta.

Table S3 Pathogenicity prediction of DSPP mutation in the study

\begin{tabular}{llll}
\hline Nucleotide changes & Amino acid changes & SIFT & PolyPhen-2 \\
\hline C.53T $>$ G & p.V18G & Deleterious & Probably damaging \\
\hline SSPP dentin
\end{tabular}

DSPP, dentin sialophosphoprotein (NM_014208).

Table S4 Mineralized calcium depositions of DGI and control

\begin{tabular}{lc}
\hline Variable & mineralized calcium deposition \\
\hline DGI & $23.32 \pm 4.27$ \\
Control & $38.77 \pm 2.05$ \\
$P$ value & 0.012 \\
\hline
\end{tabular}

DGI, dentinogenesis imperfecta.

Table S5 The expression of DSPP, ALP, and RUNX2 in patient and control cells

\begin{tabular}{lccc}
\hline Variable & DSPP & ALP & RUNX2 \\
\hline Patient & $0.38 \pm 0.18$ & $723.56 \pm 40.92$ & $2302.97 \pm 213.14$ \\
Control & $0.96 \pm 0.27$ & $710.72 \pm 92.51$ & $3075.85 \pm 36.35$ \\
P value & 0.022 & 0.866 & 0.007 \\
\hline
\end{tabular}

$D S P P$, dentin sialophosphoprotein; $A L P$, alkaline phosphatase. 